\title{
Patrick Sänger
}

\section{Neue Inschriften aus dem Domitiansdepot in Ephesos ${ }^{1}$}

Bei den nachstehenden Inschriften handelt es sich um Streufunde, die vom Ephesos Museum in Selçuk zur Aufbewahrung in das Domitiansdepot auf dem Grabungsgelände von Ephesos verbracht wurden. Fundzettel oder andere Aufzeichnungen sind nicht vorhanden, ebenso wenig wurden Inventarnummern auf den Stücken verzeichnet. Wie Nr. 2 zeigt, könnte die Herkunft der Inschriften bis nach Smyrna reichen. Als Datierungskriterium ist ausschließlich das Schriftbild heranzuziehen, welches entweder in die römische Kaiserzeit oder in die byzantinische Zeit verweist.

Das Material umfasst drei Grabinschriften (Nr. 1-3), eine davon in lateinischer, die anderen in griechischer Sprache, und eine Inschrift auf dem Bruchstück eines Sarkophags (Nr. 4). Unter den Neufunden befinden sich auch acht besonders fragmentarische Inschriften, die hier aber wegen ihres nicht mehr zu rekonstruierenden Inhaltes ausgespart bleiben, sowie auch eine fragmentarische Zweitschrift von IvE 44, der eine eigene Studie gewidmet wird ${ }^{2}$. Zusammen mit den unveröffentlichten Stücken werden auch sechs bereits bekannte Grabinschriften aufbewahrt, die von Cengiz İçten und Helmut Engelmann im Jahr 1992 unter den Nummern 25, 29, 30 und 33 sowie im Jahr 1998 unter den Nummern 10 und 11 publiziert worden waren ${ }^{3}$. Genaue Angaben zum Ort ihrer Auffindung - sofern dieser überhaupt bekannt war - haben die Editoren nicht gegeben. Zumindest für Nr. 25 und 33 sowie 10 und 11 dürfte die Herkunft aus Selçuk feststehen.

\section{1) Grabinschrift des Tiberius Plautius Agathangelus (Kaiserzeit)}

Tabula ansata aus weißem Marmor; Schriftfläche im unteren Bereich der Inschrift stark abgerieben. H 35,7 cm; B 56,8 cm; D 6 cm; Buchstabenhöhe 2,7-4,5 cm; Abb. 1.

$\begin{array}{ll}1 & \text { Dis Manibus } \\ 2 & \text { Ti(berii) Plauti(i) Agathangeli } \\ 3 & \text { vixit annis LXX } \\ 4 & \text { fecit Philetus (?) carissi[ . . ] (?) } \\ 5 & \text { os (?) [-] } \\ & {[---]}\end{array}$

\footnotetext{
${ }^{1}$ Für die Publikationsgenehmigung danken Hans Taeuber und der Verfasser der Generaldirektion für Kulturgüter und Museen, Ankara, sowie dem Direktor des Efes Müzesi, Herrn Çengiz Topal. - Zusätzlich zu den vom Österreichischen Archäologischen Institut empfohlenen Abkürzungen <http://www.oeai.at/publik/autoren.html > werden in diesem Beitrag verwendet:

IvE Inschriften griechischer Städte aus Kleinasien 11, 1 (Bonn 1979)-17, 4 (Bonn 1984) = Ephesos: IvE Ia-VIII 2.

IvSmyrna Inschriften griechischer Städte aus Kleinasien 23 (Bonn 1987)-24, 2 (Bonn 1990).

LGPN P. M. Fraser - M. Matthews, A Lexicon of Greek Personal Names I-IV (Oxford 1987-2005).

LSJ H. G. Liddell - R. Scott - H. S. Jones, A Greek-English Lexicon ${ }^{9}($ Oxford 1940).

Preisigke, WB F. Preisigke - E. Kießling, Wörterbuch der griechischen Papyrusurkunden, mit Einschluss der griechischen Inschriften, Aufschriften, Ostraka, Mumienbilder usw. aus Ägypten I-III (Berlin 1925-1931).

2 P. Sänger, Kommunikation zwischen Prätorianerpräfekt und Statthalter: Eine Zweitschrift von IvE Ia 44, Chiron 40, 2010 (in Druck).

${ }^{3}$ C. İçten - H. Engelmann, Inschriften aus Ephesos, ZPE 91, 1992, 293 Nr. 25 (= SEG 42, 1056); 294 Nr. 29 (= SEG 42, 1060) und Nr. 30 (= SEG 42, 1061); 295 Nr. 33 (= SEG 42, 1063); C. İçten - H. Engelmann, Inschriften aus Ephesos und Kolophon, ZPE 120, 1998, 89-90 Nr. 10 (= SEG 48, 1365) und Nr. 90 Nr. 11 (= SEG 48, 1366).
} 
»Den Totengöttern; (das Grabmal) des Tiberius Plautius Agathangelus; er hat siebzig Jahre gelebt; (das Grabmal) hat Philetus (?) dem liebsten [- - - gemacht.«

Der Verstorbene, Tiberius Plautius Agathangelus, ist bisher unbekannt. Der Stifter der Grabinschrift trägt wahrscheinlich den Namen Philetus (zur Lesung vgl. Kommentar zu Z. 4). Da er keinen Gentilnamen führt, dürfte er im Gegensatz zum Verstorbenen kein römischer Bürger gewesen sein. Der fehlende Vatername lässt an einen Sklaven denken. In welchem Verhältnis Agathangelus und Philetus zueinander stehen, ist unklar (vgl. hierzu auch Kommentar zu Z. 4-5).

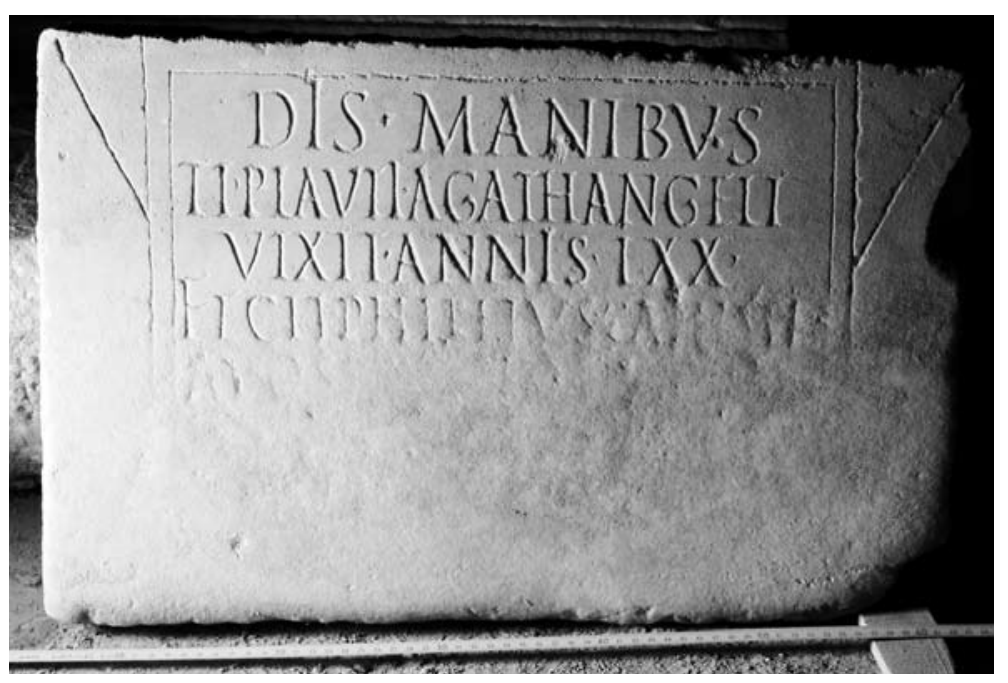

1 Grabinschrift des Tiberius Plautius Agathangelus
Z. 2. Ti(berii) Plauti(i) Agathangeli: Plautius ist ein Gentilname, der im Vergleich mit anderen römischen Gentilnamen in allen verfügbaren Quellen mit mittlerer Häufigkeit belegt ist ${ }^{4}$. Der als Kognomen verwendete griechische Personenname A $\gamma \alpha \theta \dot{\alpha} \gamma \gamma \varepsilon \lambda_{0}$, der hier im Lateinischen mit Agathangelus wiedergegeben wird, ist in Ephesos dreimal bezeugt ${ }^{5}$ und auch sonst im griechischen Sprachraum gebräuchlich ${ }^{6}$.

Z. 4. Philetus (?): Die Lesung des $t$ ist aufgrund der abgeriebenen Schriftfläche unsicher. Möglich wäre auch, Philẹ̣us zu transkribieren. Der Name $\Phi$ í $\lambda \varepsilon$ เo $\varsigma$ ist in Kleinasien aber deutlich weniger gebräuchlich als $\Phi \hat{\lambda} \lambda \eta \tau_{0} \varsigma^{7}$; in Ephesos ist er bislang gar nicht anzutreffen. Deswe-

gen wurde in der Transkription Philetus der Vorzug gegeben. Eine Person, die nur unter dem Namen Philetus (ohne Patronymikon) auftritt, belegen auch IvE 2440, 6 und 2531, 5. Eine Gleichsetzung mit unserem Grabstifter wäre in beiden Fällen rein hypothetischer Natur.

Z. 4-5. carissi[.. ] (?) | . os (?) [-]: Die Schriftspuren in Z. 5 lassen keine eindeutige Auflösung der Stelle zu. Der erste Buchstabe weist die Form eines a auf; die daneben platzierte Schräghaste könnte aber auch auf ein $m$ verweisen. Im Anschluss daran zeigen sich die Konturen eines o und eines s. Somit ergeben sich zwei Ergänzungsmöglichkeiten: Entweder carissi[mo] | ạ̣o s[uo -] oder carissi|ṃo s[-]. Die erste Variante würde das Verwandtschaftsverhältnis des Grabstifters zum Verstorbenen erhellen: Es wäre das eines Enkels und eines Großvaters. Sollte Philetus, wie weiter oben angedacht, ein Sklave gewesen sein, dann könnte Agathangelus bei dieser familiären Verbindung ein libertus gewesen sein. Problematisch wäre allerdings, -mo am Ende von Z. 4 zu ergänzen, da von beiden Buchstaben keinerlei Spuren zu erkennen sind. Zudem fehlt der Platz für eine solche Auflösung - außer man wollte annehmen, dass -mo in den Rahmen der Tabula ansata geschrieben war. Die Ergänzung carissi|ṃo s[-] wäre den Platzverhältnissen am Ende von Z. 4 immerhin angemessen. Bei dieser Variante würde sich aber ein ungewöhnlich weiter Abstand zwischen $\mathrm{m}$ und o ergeben.

\footnotetext{
${ }^{4}$ Vgl. O. Salomies, Three Notes on Roman Nomina, Arctos 32, 1998, 215, wo Plautius Gruppe D zugehört, in der Gentilnamen verzeichnet sind, die in 40-55 verschiedenen Corpora, Publikationen etc. anzutreffen sind.

${ }_{5}^{5}$ Vgl. IvE 1006, 3; 1061, 13 und B. İplikçioğlu - D. Knibbe, Neue Inschriften aus Ephesos IX, ÖJh 55, 1984, 122 Inv. 4240 (= SEG $34,1136,2)$.

${ }^{6}$ Vgl. LGPN I 1; II 2; III.A 2; B 2 und IV 1 s. v. A $\gamma \alpha \theta \dot{\alpha} \gamma \gamma \varepsilon \varepsilon \lambda$ os.

${ }^{7}$ Vgl. die unter <http://www.lgpn.ox.ac.uk/database/lgpn.php> abrufbare Suchoption (Search II) des LGPN Online, welche die Personennamen nach deren Häufigkeit in den einzelnen Volumen tabellarisch aufschlüsselt. Berücksichtigt wird hier auch das Namensmaterial Bandes V.A, der den Küstenregionen Kleinasiens von Pontos bis Ionien gewidmet ist.
} 


\section{2) Grabinschrift einer Familie mit Strafklausel (Kaiserzeit; wahrscheinlich 2./3. Jh.)}

Rechter Teil einer dicken Marmorplatte; oberer und unterer Rand erhalten; rechter Rand fast vollständig abgeschlagen; Schriftfläche stark abgerieben. H $36 \mathrm{~cm}$; B 36,8+ cm; D 11,5 cm; Buchstabenhöhe 1,5-2 cm; Abb. 2.

\begin{tabular}{|c|c|}
\hline & {$[-\mu] v \eta \mu \varepsilon \tilde{\tau} о \nu$} \\
\hline & 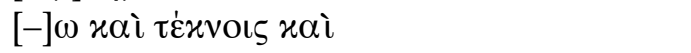 \\
\hline & 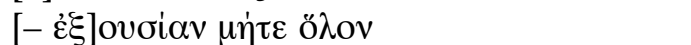 \\
\hline & {$[-] \sigma \alpha \iota \mu \eta \delta \varepsilon v \grave{\imath} \tau \varrho o ́ \tau \omega \mu \eta$} \\
\hline & 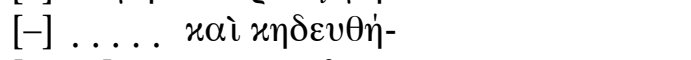 \\
\hline & 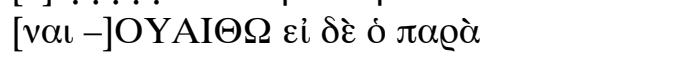 \\
\hline & 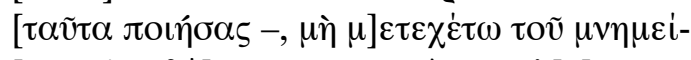 \\
\hline & 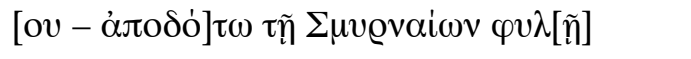 \\
\hline
\end{tabular}

»Grabmal [- - - ] und den Kindern und [- - - B Befugnis weder ganz [- - -] keiner Weise [- - -] und bestattet zu werden [- - - ] wenn aber, soll der, der zuwider [handelt - - -], nicht teilhaben an dem Grabmal [- - - sondern] der Phyle der Smyrnäer zahlen [- - - ].«

Es liegt eine fragmentarische und aufgrund der abgeriebenen Schriftfläche schwer lesbare Grabinschrift vor. Der Textverlust auf der linken Seite ist wegen der oftmals vielfältigen Ergänzungsmöglichkeiten schwer abzuschätzen und daher kaum genau festzulegen. Außerdem scheint das Formular an manchen Stellen von den bisher bekannten Formen abzuweichen. Wenigstens bleibt festzuhalten, dass der erhaltene Teil weniger als die Hälfte der Inschrift überliefern dürfte (vgl. Kommentar zu Z. 1-3 und 3).

Die Provenienz des Stückes könnte Ephesos oder Smyrna sein. Die Ausdrucksweise der Inschrift hat Parallelen in beiden Siedlungsgebieten, wenngleich für die Formulierung in den Z. 6-8 hauptsächlich smyrnäische Grabinschriften als Vergleichsbeispiele heranzuziehen sind. Auch die bislang unbekannte $\Sigma \mu v \varrho v \alpha i \omega v$ $\varphi v \lambda \eta ́$ (Z. 8) liefert keinen sicheren Hinweis auf die Herkunft der Inschrift. Wie die $\varphi v \lambda \grave{\eta}$ 'E

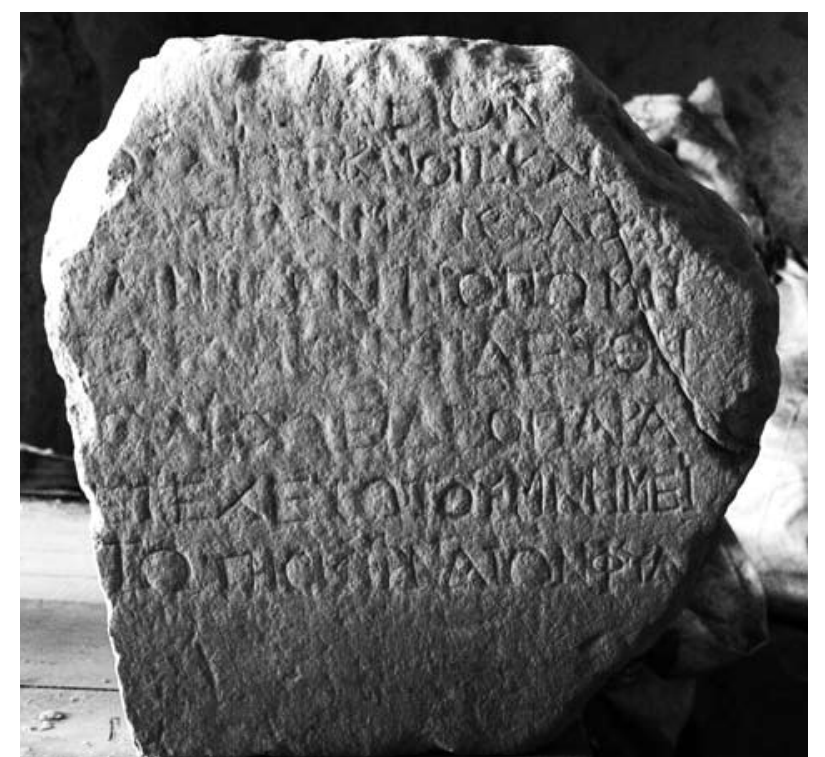

2 Grabinschrift einer Familie mit Strafklausel Phyle gewesen $\operatorname{sein}^{8}$. Bei der $\Sigma \mu v \varrho v \alpha i \omega v$ $\varphi v \lambda \eta$ könnte es sich aber genauso gut um eine ephesische Phyle handeln, deren Existenz hier das erste Mal bezeugt ist; zumindest gibt es in Ephesos auch eine Chiliastye namens $\Sigma \mu v \varrho v \alpha i{ }^{9} \iota^{9}$.

Aus $\tau \dot{\varepsilon} x$ voı $\varsigma$ in Z. 2 erschließt sich, dass das Grab für eine Familie errichtet wurde. Der Name des Stifters

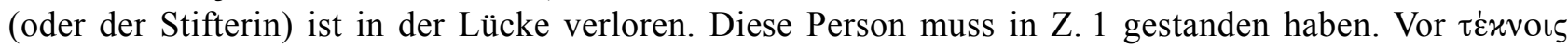
könnte der Gatte (oder die Gattin) des Stifters (oder der Stifterin) angeführt worden sein (vgl. Kommentar zu Z. 1-3). In Z. 3 setzt die Strafklausel ein, die widerrechtlichen Zugriff auf das Grab untersagt und gegen die Bestimmungen handelnde Personen mit einer Strafe belegt.

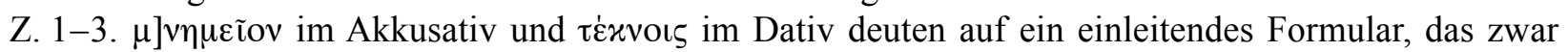
nicht in Grabinschriften aus Ephesos, dafür aber in solchen aus Smyrna mit einiger Häufigkeit auftritt. In Analogie zu diesen Parallelen könnte der Text folgendermaßen zu rekonstruieren sein: [Grabstifter(in)

\footnotetext{
${ }^{8} \mathrm{Zu}$ den ephesischen Phylen und Chiliastyen s. D. Knibbe, Der Staatsmarkt. Die Inschriften des Prytaneions. Die Kureteninschriften und sonstige religiöse Texte, FiE 9, 1, 1 (Wien 1981) 107-109 und bes. Anm. 256.

${ }_{9}^{9}$ Die Phylenzugehörigkeit der Chiliastye $\Sigma \mu v \varrho v \alpha$ ĩo ist unklar; vgl. Knibbe (Anm. 8) 109.
} 


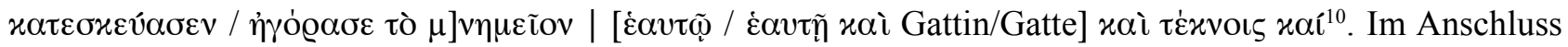
daran dürften in der Lücke von Z. 3 noch weitere Angehörige der familia genannt worden sein (etwa Enkel

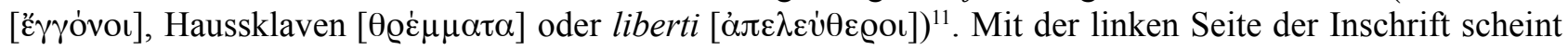
somit mehr Text verloren zu sein, als sich auf der rechten Seite erhalten hat (vgl. auch Kommentar zu Z. 3).

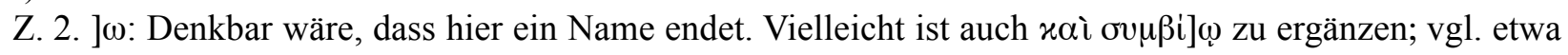
IvSmyrna 197, 7 und 206, $2^{12}$.

Z. 3-8. Diese Zeilen werden von der Strafklausel der Grabinschrift eingenommen. An manchen Stellen erlauben die Parallelen eine Rekonstruktion des Formulars.

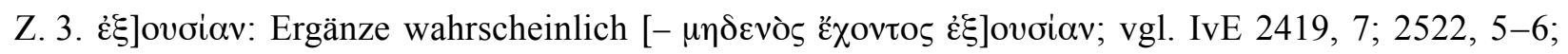
IvSmyrna 199, 5-6; 213, 5-6; 268, 4-5. Mit diesen Worten wird die Strafklausel für gewöhnlich eingeleitet. Diese stereotype Konstruktion vermittelt einen sehr schönen Eindruck über das Ausmaß des Textverlustes, der in jedem Fall mehr als die Hälfte der Grabinschrift umfasst hat (vgl. auch Kommentar zu Z. 1-3).

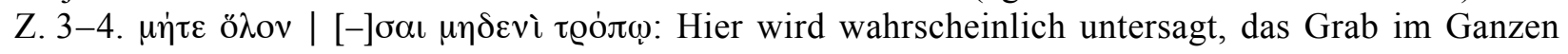

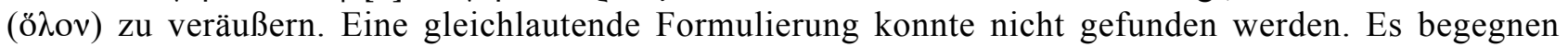

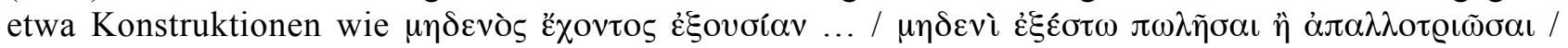

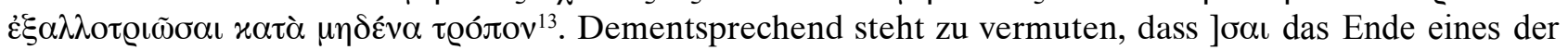
angezeigten Verben ist.

Z. 4-5. $\mu \eta \mid[-]$. . . . . : Was der genaue Inhalt dieses Passus war, bleibt verborgen. Der vorangehenden Satzkonstruktion folgend sind die Ergänzung $\mu \eta \dot{\mid} \mid[\tau \varepsilon$ und die Angabe eines weiteren Verbotes anzunehmen. Da zuvor der Verkauf des gesamten Grabes untersagt wurde, könnte an dieser Stelle auch dessen partielle Veräußerung Inhalt der Strafklausel gewesen sein. Eine vergleichbare Formel lautet in IvSmyrna 252:

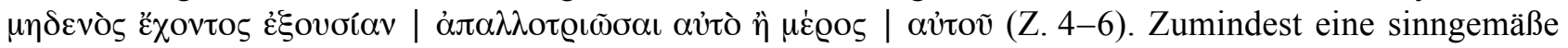
Formulierung wäre in unserem Fall gut denkbar, deren Abschluss vor xai in Z. 5 zu vermuten ist. Außer einiger marginaler Überreste von fünf Buchstaben ist hier aber nichts mehr zu erkennen.

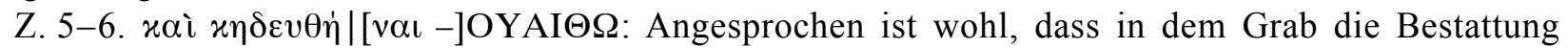
von anderen als den genannten Personen nicht gestattet ist. Ähnliche Verbote finden sich in folgenden Grabinschriften aus Ephesos und Smyrna ${ }^{14}$ :

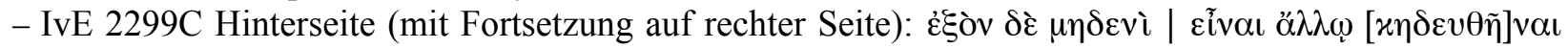

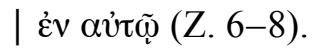

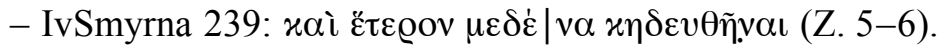

Eine mit unserem Text übereinstimmende Wortstellung ist nicht festzustellen. Darauf hingewiesen sei, dass statt $¥ \eta \delta \varepsilon \dot{\varepsilon} \varepsilon v$ auch die Verben $\theta \dot{\alpha} \pi \varepsilon \iota v$ oder $\theta \varepsilon \tilde{\imath} v \alpha \iota$ verwendet wurden, so z. B. in IvSmyrna 252, 6 (iे

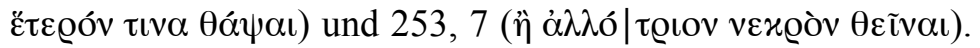

Die Auflösung der Buchstabenfolge OYAI $\Omega \Omega$ muss offenbleiben. Da diese Stelle der Inschrift besonders stark abgerieben ist, kann die Richtigkeit der Lesung nicht garantiert werden. Syntaktisch gehört der hier

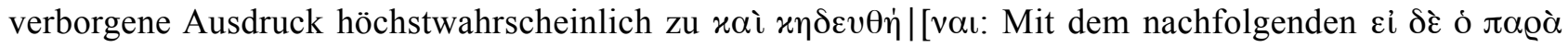
beginnt nämlich ein neuer Satzteil.

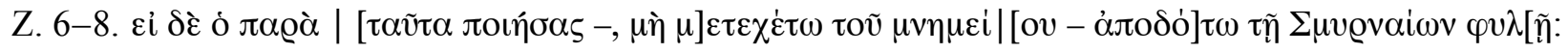
Damit wird auf die Konsequenzen aus dem widerrechtlichen Verkauf des Grabes und der Bestattung nicht vorgesehener Personen in diesem hingewiesen: 1) unrechtmäßige Inbesitznahme; 2) Bezahlung einer Strafsumme.

\footnotetext{
${ }^{10}$ Vgl. z. B. IvSmyrna 197, 1-9; 201, 2-5; 206, 1-3; 219, 1-4; 222; 233, 1-5; 237, 1-7; 252, 1-4; 290, 1-6; 319; 322; 328. Auch die

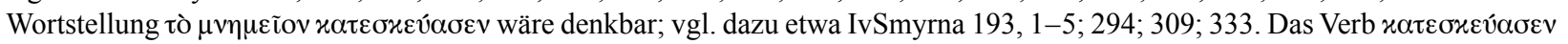
kann im Übrigen auch entfallen; so in IvSmyrna 215, 1-4.

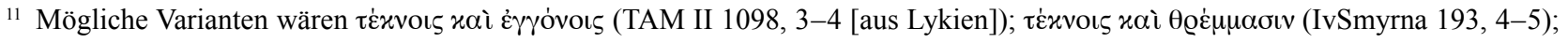

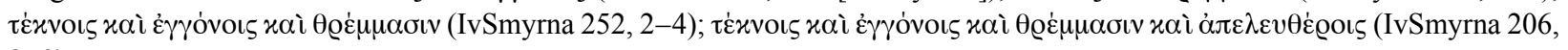
2-3).

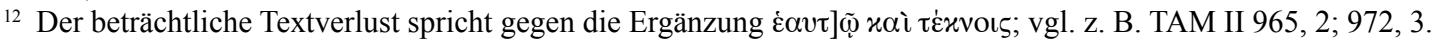

13 Vgl. z. B. IvSmyrna 213, 5-9; 242, 7-10 sowie auch IvE 2419, 8-9 (ergänzt).

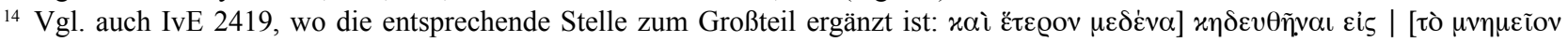
(Z. 6-7).
} 


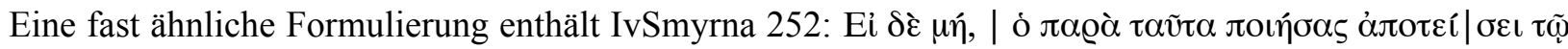

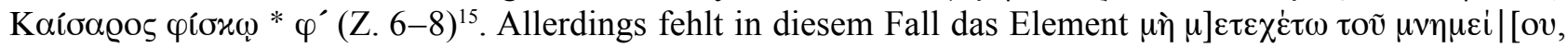
das in unserer Inschrift noch vor der Bekanntgabe der Strafsumme eingeschoben ist. Vorhanden ist es dagegen

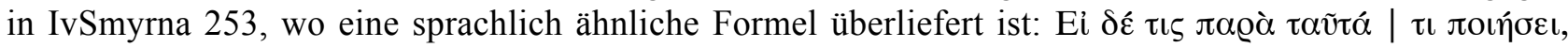

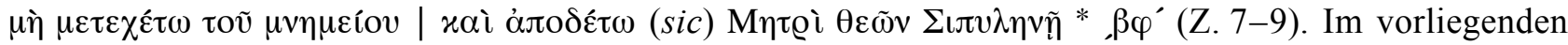
Fall dürfte es sich demnach um eine Mischform aus beiden Varianten gehandelt haben; $\mu$ ๆ́ wurde nach

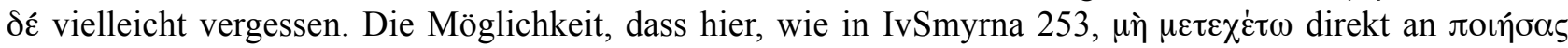
anschließt, ist eher unwahrscheinlich, denn vor allem die ersten drei Zeilen deuten auf einen Textverlust hin, der mit der in Frage stehenden Formel allein kaum zu füllen wäre (vgl. Kommentar zu Z. 1-3 und 3).

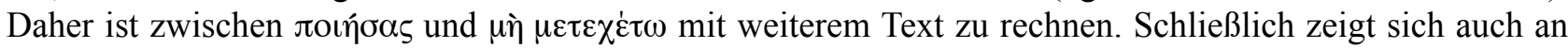
Z. 8, dass die Länge der in IvSmyrna 253 belegten Formel mit den vorliegenden Platzverhältnissen nicht in

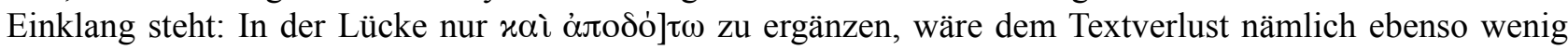
angemessen.

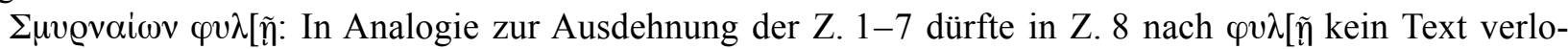
ren sein. Die verschollene Angabe der Strafsumme ist daher in einer weiteren Zeile zu vermuten, die aber vor der Bruchkante endete und damit keine Spuren hinterlassen hat. Zu zahlen war die Strafsumme an die smyräische Phyle, über die vorläufig kein weiterer Beleg unterrichtet und deren Lokalisierung offenbleiben muss (vgl. dazu oben in der Einleitung).

\section{3) Grabinschrift mit unklarem Inhalt (byzantinische Zeit)}

Rechter Abschluss einer Marmorplatte; oberer, unterer und rechter Rand erhalten. H 38,5 cm; B 28,9 cm; D 2,2 cm; Buchstabenhöhe 1,5-3 cm; Abb. 3.

\begin{tabular}{|c|c|}
\hline & 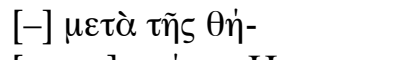 \\
\hline & 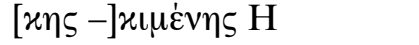 \\
\hline & {$[-] \theta \dot{\eta} x \eta \varsigma \gamma \varepsilon \gamma-$} \\
\hline & [-] غ̇xĩvo KAIP \\
\hline & 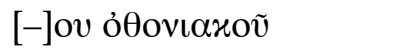 \\
\hline & 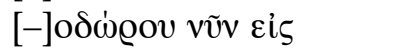 \\
\hline & {$[-] \delta ı x \varepsilon \dot{\omega} \mu \alpha \tau \omega \vee$ METH } \\
\hline & 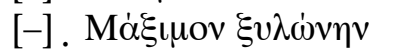 \\
\hline & 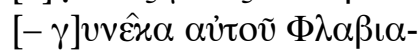 \\
\hline & {$[-\varkappa \lambda \eta \varrho o] y o ́ \mu \omega v \alpha v ่ \tau-$} \\
\hline & {$[\tilde{\omega} v-\tau \tilde{\omega}] v \xi \omega \dot{\omega} \tau \omega(\nu)$} \\
\hline
\end{tabular}

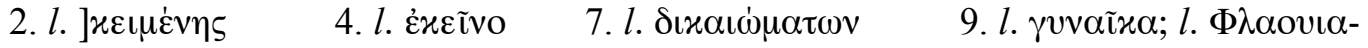

Das Fragment enthält eine Grabinschrift, deren Verständnis Schwierigkeiten bereitet. Der Text lässt sich aus den vorhandenen Parallelen nicht erschließen. Daher bleibt auch die ursprüngliche Zeilenlänge im Dunkeln.

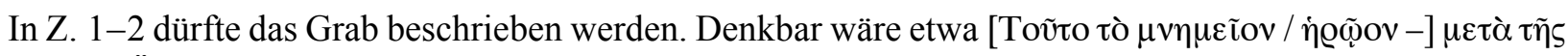
$\theta \dot{\eta} \mid[\varkappa \eta \varsigma$ o. Ä.; der Gebrauch der Präposition $\mu \varepsilon \tau \alpha ́$ an dieser Stelle ist sonst in keiner weiteren Grabinschrift

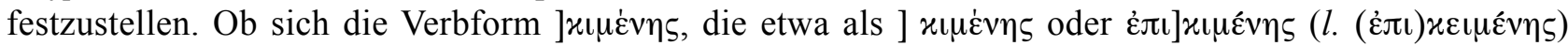
aufzulösen sein wird, auf $\theta \dot{\eta} \mid[x \eta \varsigma$ oder einen nachfolgenden Begriff bezieht, ist kaum mit Sicherheit festzulegen. In den Grabinschriften begegnen beide Varianten. In der Inschrift ÖJh 55, 1984, 117 Inv. 4195

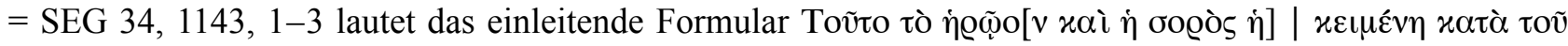

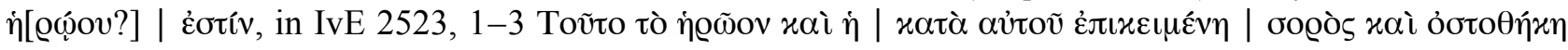

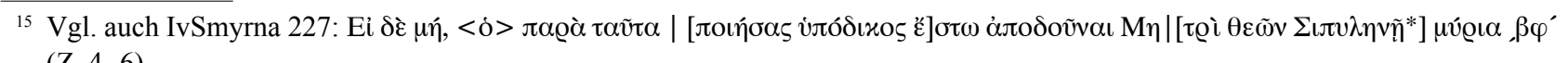
(Z. 4-6). 


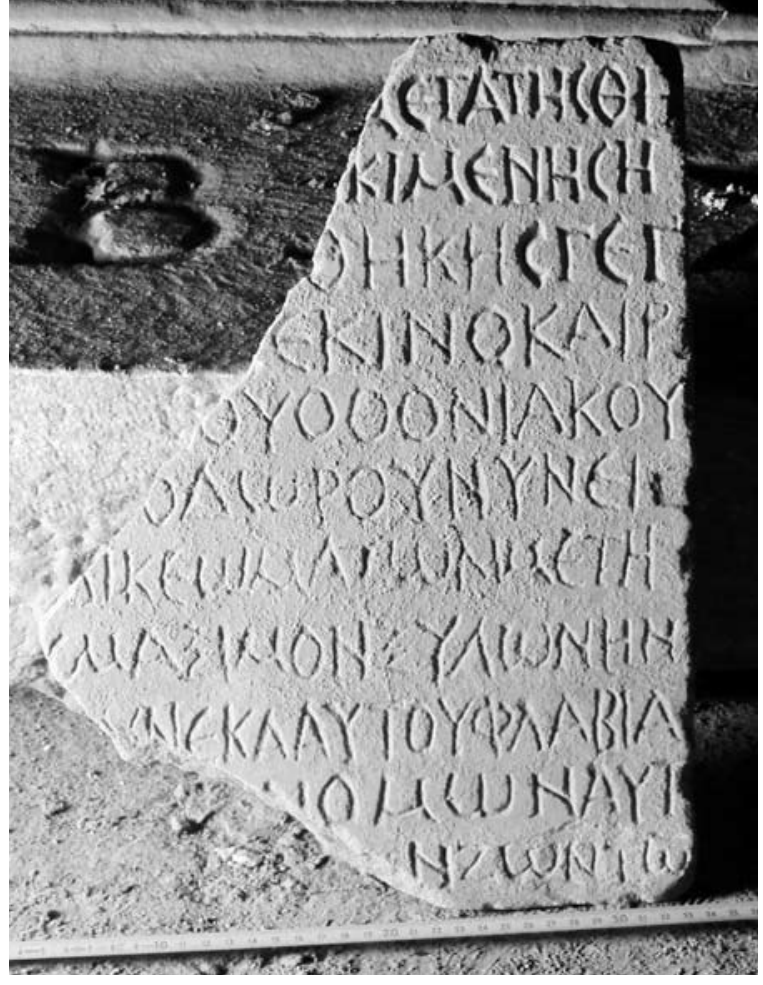

3 Grabinschrift mit unklarem Inhalt

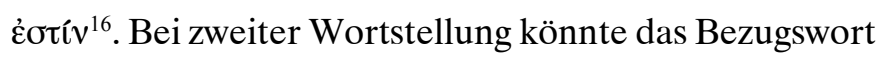
in unserem Fall mit H, dem letzten Buchstaben in Z. 2, begonnen haben. Vielleicht ist danach noch ein weiteres Objekt Teil der Konstruktion, dessen Ende wir mit ] $\theta \dot{\eta}-$ xๆ vor uns haben. Bei diesen Überlegungen stellt sich jedoch die Frage nach der Sinnhaftigkeit einer solchen Formulierung, denn $\theta \dot{\eta} \mid[x \eta \varsigma$ in Z. 1 würde außerhalb der Partizipialkonstruktion bleiben. Somit würde eine

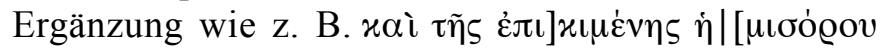

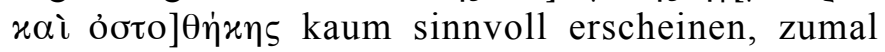
sie aufgrund der unsicheren Platzverhältnisse rein hypothetischen Charakter hätte. Dasselbe Problem stellt

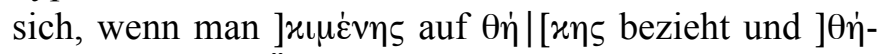
xๆ betrachtet: Die Beziehung zwischen beiden Begriffen müsste völlig offenbleiben. In Analogie zu den zitierten Formularen erschiene schließlich auch die Position von $\gamma \varepsilon \gamma[$, das den Beginn einer Verform darstellt, unpassend stattdessen würde man eher غ̇oтív erwarten.

Ebenso fraglich muss die inhaltliche Bedeutung der Wortfolge [-] غ̇xĩvo KAIP in Z. 4 bleiben. Danach haben

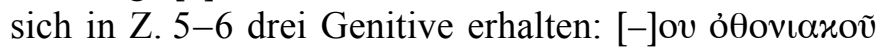
| [-]odó@ov. Möglicherweise sind an dieser Stelle der oder die Inhaber des Grabes genannt. Die Endungen ]ov

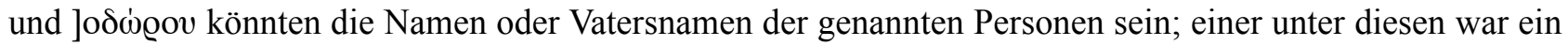
oӨovıaxóร, ein »Leinenhändler« (vgl. dazu Kommentar zu Z. 5).

Der Inhalt des anschließenden Passus ist gänzlich rätselhaft. Den Ausdruck võv ciऽ (Z. 6) kann ich mir ebenso wenig erklären wie die plötzliche Erwähnung von »Urteilen«, »Rechtsansprüchen«, »Dokumenten«

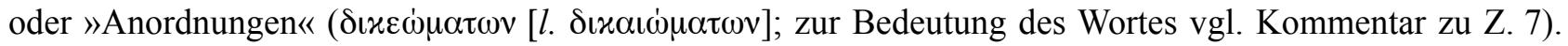
Bei Urteilen und Anordnungen könnte man an die Tätigkeit eines weltlichen Richters denken oder das

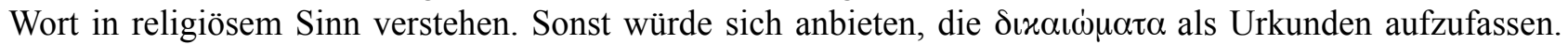
Aufgrund des unklaren Kontextes ist es m. E. aber nicht möglich, den in Frage stehenden Begriff auf eine der genannten Varianten zu beschränken. In Z. 8-9 werden dann noch einmal Personen angeführt. Es ist von

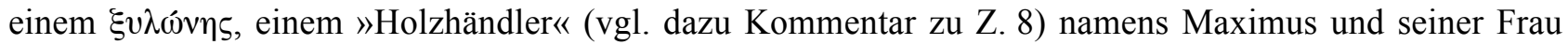
Flavia(ne) die Rede. Ihre Angabe erfolgt im Akkusativ, weswegen - zumindest angesichts des erhaltenen Textes - ein direkter Zusammenhang mit $x \lambda \eta \varrho o] y o ́ \mu \omega v ~(Z .10)$ nicht gegeben scheint. Von entscheidender Bedeutung für das inhaltliche Verständnis der Grabinschrift ist die Frage, in welcher Funktion oder zu welchem Zweck Maximus und seine Frau genannt werden. Ein bestimmtes Rechtsverhältnis zu dem Inhaber des Grabes darf wohl vorausgesetzt werden. Waren es vielleicht Maximus und seine Frau, die das aktuelle Nutzungsrecht an der Begräbnisstätte besaßen? Und wie ist dann die Stellung der in Z. 5-6 verzeichneten Person(en) einzustufen? Ohne einschlägige Parallelen und zusammenhängende Textpassagen ist darüber freilich nur zu spekulieren.

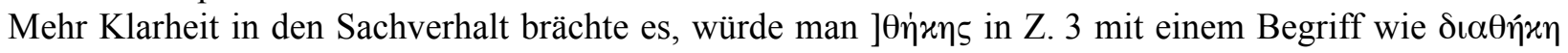

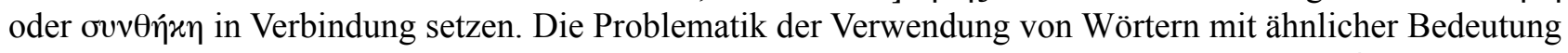

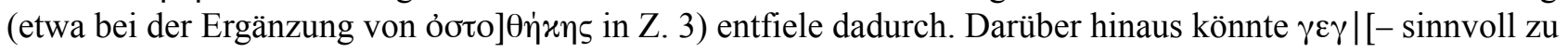
$\gamma \varepsilon \gamma \mid[\varrho \alpha \mu \mu \varepsilon ́ v \eta \varsigma$ ergänzt werden. Daraus wäre eine urkundlich verbriefte Willensäußerung, möglicherweise in testamentarischer Form, abzuleiten, als deren Urheber der oder die Personen in Z. 5-6 angesehen werden könnten. Weiters könnte man mutmaßen, dass in dieser Urkunde die Nutzungsrechte an dem Grab an Maximus und seine Frau übertragen worden waren. Ohne dieses Interpretationsmodell auch nur hinlänglich beweisen

${ }^{16} \mathrm{Zu}$ dieser Wortstellung vgl. auch IvE 2200BIII, 1-2; 2222A, 1-3; C, 1 und İçten - Engelmann (Anm. 3:1992) 289 Nr. 15 (= SEG $42,1048,3-4)$. 
zu können, würde es trotz der angezeigten Unsicherheiten das inhaltliche Verständnis der fragmentarischen Inschrift doch weit vorantreiben.

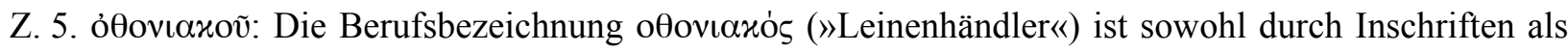
auch Papyri überliefert ${ }^{17}$.

Z. 6. ]odó@ov: Für die Auflösung des hier verborgenen Namens gibt es freilich viele Varianten; am

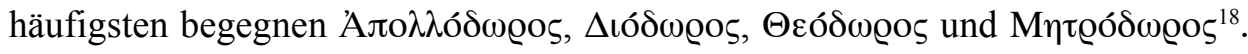

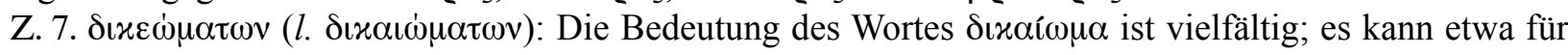
$»$ Urteil«, »Rechtsanspruch«, »Dokument«, »Beweisurkunde« oder »Anordnung « stehen ${ }^{19}$.

METH: Vielleicht handelt es sich um eine Aorist- oder Perfektform von $\mu \varepsilon \tau \alpha \lambda \lambda \dot{\alpha} \sigma \sigma \varepsilon เ v(\mu \varepsilon \tau \eta \lambda \lambda \alpha \xi-$ / $\mu \varepsilon \tau \alpha \lambda \lambda \alpha \chi-)$.

Z. 8. ]. : Die Linienführung deutet ein Sigma an.

$\xi v \lambda \omega \omega v \eta v$ : Die Berufsbezeichnung $\xi v \lambda \dot{\omega} v \eta \varsigma$ ist ein Hapaxlegomenon. Einen ähnlichen Ausdruck stellt vi $\omega_{\text {ovn }}$ dar, der bislang nur in SEG 37, 100. 103. 141 (Oropos, ca. 330 v. Chr.) belegt ist. Die Wortbildung

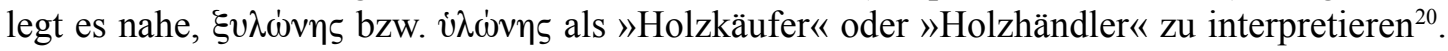

Z. 9. $\Phi \lambda \alpha \beta \iota \alpha \mid[-:$ Zu ergänzen ist entweder $\Phi \lambda \alpha \beta i ́ \alpha \mid[v$ oder $\Phi \lambda \alpha \beta \iota \alpha \mid[v \eta ́ v$.

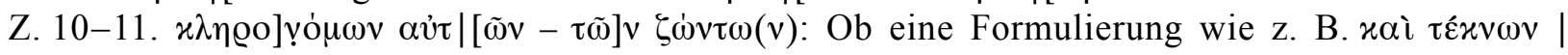

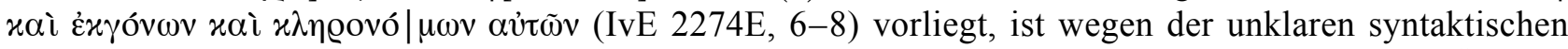
Beziehung zwischen $x \lambda \eta$ ¡o]

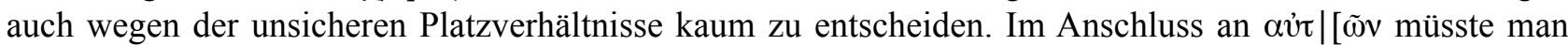
bis $\tau \tilde{\omega}] v \xi \dot{\omega} v \tau \omega(v)$ noch mit Text rechnen.

$\zeta \dot{\omega} v \tau \omega(v)$ : Über dem zweiten Omega befindet sich ein Kürzungsstrich.

\section{4) Sarkophaginschrift des Lollios Paris (Kaiserzeit; wahrscheinlich 2./3. Jh. n. Chr.)}

H 8+ cm; B 38+ cm; D 6,5+ cm; Buchstabenhöhe 1,5-2 cm; über dem Schriftfeld sind Reste eines Girlandenschmucks zu erkennen; Abb. 4.

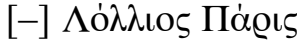

$»[--$ - $]$ Lollios Paris«

Das Fragment war Teil eines Sarkophags. Die genannte Person ist bislang unbekannt. Bei ihr dürfte es sich um den Verstorbenen handeln, wenngleich man dessen Angabe eher im Genitiv erwarten würde; vgl. z. B. IvE 3308. Der Name Пó@ıs wäre für Ephesos das erste Mal belegt.

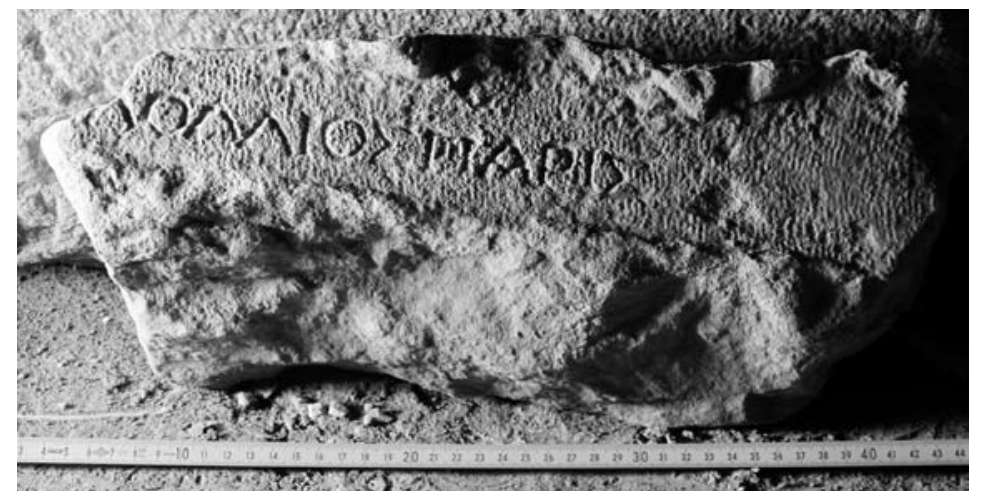

4 Sarkophaginschrift des Lollios Paris

Mag. Dr. Patrick Sänger

Universität Heidelberg, Zentrum für Altertumswissenschaften, Institut für Papyrologie, Marstallstraße 6, D-69117 Heidelberg

E-Mail: patrick.saenger@zaw.uni-heidelberg.de

Abbildungsnachweis: Alle Abb.: H. Taeuber und P. Sänger; C ÖAI.

\footnotetext{
${ }^{17}$ Vgl. LSJ 1200 und Preisigke, WB II 152 s. v. ỏӨovıaxós.

${ }_{18}$ So die Ergebnisse der oben in Anm. 7 erläuterten Suchoption des LGPN Online.

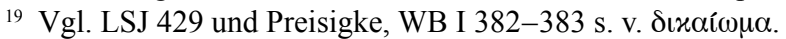

${ }^{20}$ Vgl. SEG 37, 100 und die editio princeps dieser Inschrift, wo M. K. Langdon, An Attic Decree Concerning Oropos, Hesperia 56,

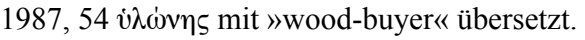


